\title{
Effect of physical and chemical doping on optical spectra of SWNT's
}

\author{
K. Kamarás ${ }^{* \dagger}$, H.Hu ${ }^{\dagger}$, B. Zhao ${ }^{\dagger}$, S. Niyogi ${ }^{\dagger}$, M.E. Itkis ${ }^{\dagger}$ and R.C. Haddon ${ }^{\dagger}$ \\ ${ }^{*}$ Research Institute for Solid State Physics and Optics, Hungarian Academy of Sciences, \\ P. O. Box 49, Budapest, H-1525, Hungary \\ ${ }^{\dagger}$ Center for Nanoscale Science and Engineering, Departments of Chemistry and Chemical and \\ Environmental Engineering, University of California, Riverside, CA 92521-0403, U.S.A.
}

\begin{abstract}
We discuss the use of far-infrared spectroscopy in the characterization of doped and functionalized nanotube derivatives.
\end{abstract}

\section{INTRODUCTION}

Optical spectroscopy is one of the most widely used methods in characterization of carbon nanotubes. Recently, it was demonstrated that absorption and especially fluorescence studies can detect individual nanotubes and identify them by chirality index [1]. Most studies concentrated on the NIR/VIS spectral range where transitions between van Hove singularities occur: based on these observations, selectivity by semiconducting/metallic character was reported both for ionic doping [2, 3] and covalent functionalization [4]. Relatively less attention was devoted to the low-frequency part of the spectrum. Here we want to emphasize the importance of far-infrared measurements as a sensitive indicator of intrinsic charge carriers in metallic tubes and extrinsic carriers in doped materials.

\section{EXPERIMENTAL}

We used arc-produced nanotube powders for the doping experiments and HiPCO nanotubes for functionalization. Details of functionalization are described in Ref. [5]. Nitric acid doping was performed under ambient conditions by exposing the films to saturated $\mathrm{HNO}_{3}$ atmosphere for one hour.Thin films for transmission measurements were prepared on silicon and sapphire substrates from DMF suspensions using an airbrush [6]. Spectroscopic measurements were performed on a Bruker 120HR FTIR instrument working in the far and mid-infrared (silicon samples), and a Varian Cary 5000 NIR/VIS/UV dispersive spectrometer (sapphire samples). Transmission spectra were converted to absorbance without further correction and scaled together in the overlap region. 


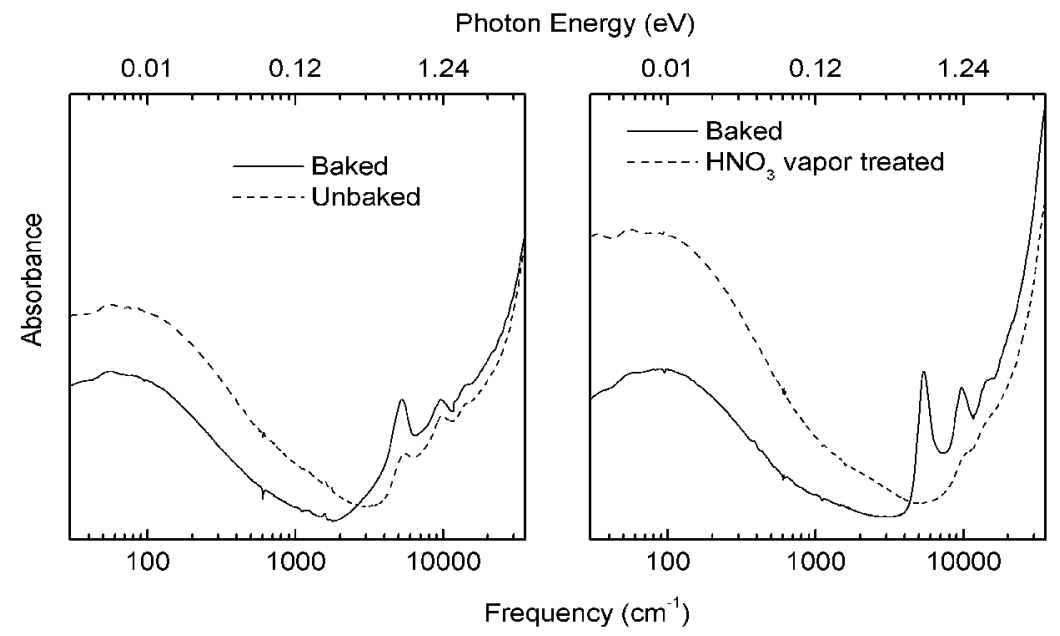

FIGURE 1. Change in absorbance of SWNT films on baking of $\mathrm{HNO}_{3}$ purified sample (left panel), treating $\mathrm{HCl}$ purified sample with $\mathrm{HNO}_{3}$ vapor (right panel). Note the logarithmic frequency scale to emphasize the far-infrared region.

\section{RESULTS AND DISCUSSION}

We present two examples for ionic doping and chemical functionalization, respectively: $\mathrm{HNO}_{3}$ treatment (which occurs during standard steps of purification) and covalent functionalization by dichlorocarbene. Figure 1 shows optical spectra taken during two separate experiments: removal of $\mathrm{HNO}_{3}$ from purified as-prepared SWNT's and intentional doping of another SWNT sample which was previously purified by $\mathrm{HCl}$ and then annealed at $450^{\circ} \mathrm{C}$ in vacuum. The effect of nitric acid is twofold: introduction of $-\mathrm{COOH}$ groups to the ends and defects of nanotubes, and increasing the concentration of mobile charge carriers by removing electrons from the filled states of semiconducting nanotubes. The latter process is similar to nitrate intercalation into graphite [7]. The addition of free carriers is reflected in the low-frequency absorption, but there are also more subtle changes in the interband transitions which have been observed before. In the purified sample (dashed line in the left panel of Fig. 1), the interband transitions are still discernible, but in the vapor-treated sample (dashed line in the right panel) they disappear. This difference, together with the higher far-infrared intensity in the vapor-treated material, indicates that the $\mathrm{HNO}_{3}$-purified sample has a lower carrier concentration than the vapor-treated one. As in graphite, several equilibrium doped phases with varying dopant concentration seem to exist $[2,3]$. The far-infrared oscillator strength change thus corroborates the model outlined by Kazaoui et al. [2] for the sequence of doping, also in the case of nitrate.

A very different result emerges when introducing covalent side groups to carbon 
nanotubes. This is shown in Figure 2, left panel. Dichlorocarbene addition transforms $\mathrm{sp}^{2}$ carbon atoms to $\mathrm{sp}^{3}$ carbons, and thus effectively interrupts the conjugation of double bonds on the tube surface. As a consequence, the one-dimensional electronic structure collapses, causing both interband transitions and free-carrier absorption to decrease and eventually disappear at high enough sidegroup concentration. The farinfrared region in this case reflects the effect of functionalization on metallic tubes. Selective functionalization of metallic tubes has been demonstrated by Strano et al. [4] who monitored the $\mathrm{M}_{11}$ interband transition and explained their findings along similar lines. Although we do not detect signs of selectivity in this reaction (because of the different mechanism), the present results support that explanation.

The right panel of Figure 2 illustrates the effect of annealing on dichlorocarbene functionalized material. Raman spectra of this substance [5] reveal that on annealing, both the $\mathrm{C}-\mathrm{Cl}$ vibrational transition and the graphitic D-band decrease in intensity, indicating formation of intertube bonds with $\mathrm{sp}^{2}$ carbons. However, no change occurs in the region of free carriers, proving that the one-dimensional collective electron system does not recover.

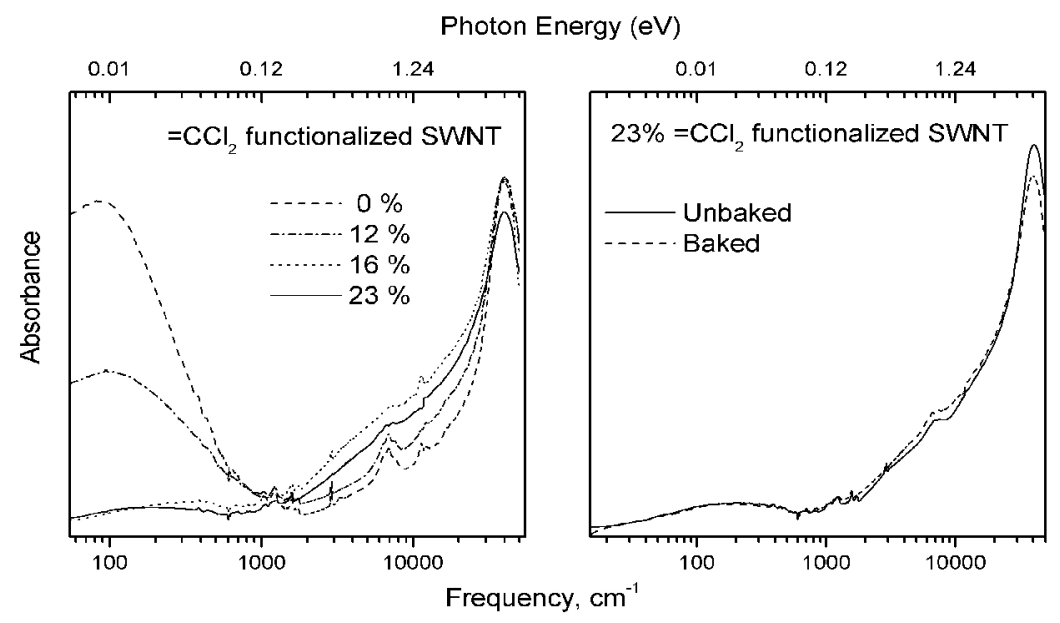

FIGURE 2. Left panel: Absorbance of HiPCO films functionalized with dichlorocarbene at different functionalization levels. Right panel: Absorbance of the highest functionalization dichlorocarbene product before and after thermal treatment at $450 \mathrm{C}$.

\section{CONCLUSIONS}

Far-infrared spectra give valuable information on both intrinsic free carriers in metallic nanotubes and extrinsic ones introduced by intentional or unintentional doping. While ionic doping increases the number of free carriers and thus the far-infrared absorption, 
covalent functionalization destroys the collective electronic system by localizing the carriers. The effect is much more sizable than the changes in the $\mathbf{M}_{11}$ transition, therefore extending the spectral range towards low frequencies can be very useful in chiralitydependent studies.

\section{ACKNOWLEDGMENTS}

This work was supported by the following grants: DARPA DMEA90-02-2-0216, OTKA T 034198.

\section{REFERENCES}

1. Bachilo, S. M., Strano, M. S., Kittrell, C., Hauge, R. H., Smalley, R. E., and Weissman, R. B., Science, 298, 2361 (2002).

2. Kazaoui, S., Minami, N., Jacquermin, R., Kataura, H., and Achiba, Y., Phys. Rev. B, 60, 13339 (1999).

3. Hennrich, F., Wellmann, R., Malik, S., Lebedkin, S., and Kappes, M. M., Phys. Chem. Chem. Phys., 5, 178 (2003).

4. Strano, M. S., Dyke, C. A., Usrey, M. L., Barone, P. W., Allen, M. J., Shan, H., Kittrell, C., Hauge, R. H., Tour, J. M., and Smalley, R. E., Science, 301, 1519 (2003).

5. Hu, H., Zhao, B., Hamon, M. A., Kamarás, K., Itkis, M. E., and Haddon, R. C., J. Am. Chem. Soc., 125, 14893 (2003).

6. Itkis, M. E., Niyogi, S., Meng, M. E., Hamon, M. A., Hu, H., and Haddon, R. C., Nano Lett., 2, 155 (2002).

7. Ubbelohde, A. R., Proc. Roy. Soc. London Ser. A, 304, 25 (1968). 